\title{
ON THE SOLUTION OF STOCHASTIC OPTIMIZATION PROBLEMS IN IMPERFECT INFORMATION REGIMES
}

\author{
Hao Jiang \\ Industrial and Enterprise Systems Engineering \\ University of Illinois \\ Urbana, IL 61801, USA
}

\author{
Uday V. Shanbhag \\ Industrial and Manufacturing Engineering \\ Pennsylvania State University \\ University Park, PA, 16803, USA
}

\begin{abstract}
We consider the solution of a stochastic convex optimization problem $\mathbb{E}\left[f\left(x ; \theta^{*}, \xi\right)\right]$ in $x$ over a closed and convex set $X$ in a regime where $\theta^{*}$ is unavailable. Instead, $\theta^{*}$ may be learnt by minimizing a suitable metric $\mathbb{E}[g(\theta ; \eta)]$ in $\theta$ over a closed and convex set $\Theta$. We present a coupled stochastic approximation scheme for the associated stochastic optimization problem with imperfect information. The schemes are shown to be equipped with almost sure convergence properties in regimes where the function $f$ is both strongly convex as well as merely convex. Rate estimates are provided in both a strongly convex as well as a merely convex regime, where the use of averaging facilitates the development of a bound.
\end{abstract}

\section{INTRODUCTION}

Increasingly, optimization problems need to be solved in uncertain networked regimes. A challenge in developing the associated distributed protocols lies in ensuring that all agents have access to system parameters, particularly when such parameters are essential for evaluating agent-specific cost functions. However, such parameters may be unavailable and require learning through a separate process. Historically, this question has been addressed via a two-step, and in effect, a serial approach. The first step requires the learning of such parameters by possibly fitting a model to a set of samples collected off-line, a problem that falls within the purview of statistical learning (Hastie, Tibshirani, and Friedman 2001). Given an estimate of such parameters, optimization algorithms can be subsequently applied. Unfortunately, in many dynamic settings complicated by streaming data and the need for online decision-making, one cannot impose such a separation in these processes and both optimization and learning need to be carried out simultaneously.

In contrast with a serial approach, we consider an avenue where the learning and optimization problem are jointly resolved and consider a more general class of coupled stochastic optimization problems. Of these, the primary problem is the following parameterized stochastic optimization problem:

$$
\min _{x \in X} f\left(x ; \theta^{*}\right) \triangleq \mathbb{E}\left[f\left(x ; \theta^{*}, \xi\right)\right],
$$

where $X \subseteq \mathbb{R}^{n}$ is a closed and convex set, $\xi: \Omega \rightarrow \mathbb{R}^{d}$ is a $d$-dimensional random variable, $f: X \times \mathbb{R}^{d} \times \mathbb{R}^{m} \rightarrow$ $\mathbb{R}$ is a real-valued function. Suppose the associated probability space is denoted by $\left(\Omega, \mathscr{F}_{x}, \mathbb{P}_{x}\right)$ and $\theta^{*}$ is an unknown parameter vector that may be learnt through the solution of a second stochastic optimization problem using a collection of already collected samples as given by the following:

$$
\min _{\theta \in \Theta} g(\theta) \triangleq \mathbb{E}[g(\theta ; \eta)]
$$

where $\Theta \subseteq \mathbb{R}^{m}$ is a closed and convex set, $\eta: \Lambda \rightarrow \mathbb{R}^{p}$ is a random variable on a probability space $\left(\Lambda, \mathscr{F}_{\theta}, \mathbb{P}_{\theta}\right)$. Furthermore, $g: \Theta \times \mathbb{R}^{p} \rightarrow \mathbb{R}$ is a real-valued function. Given the generality of the model, a range of applications emerge, a subset of which we describe next. 
(i) Stochastic optimization problems with imperfect information: As mentioned earlier, a key motivation for studying the proposed problem emerges from imperfect information stochastic optimization problems. In such settings, the decision-maker may not have access to specific system-level parameters; such parameters may take the form of specific structural or thermal characteristics or may represent market-level characteristics such as demand, prices or elasticities. The prescribed model assumes relevance when such parameters can be "learnt" by solving a suitably defined problem.

(ii) Coupled stochastic optimization problems: In many networked settings, a collection of agents may be motivated by prescribed protocols. However, agent-specific decisions may rely on the outcome of a distinct consensus or optimization process over a related network, not unlike the interactions in a layered or hierarchical networked multiagent system. An instance of such a problem arises in the context of a smart grid where macro and micro grids interact. Here, $\theta^{*}$ may take the form of demand response or distributed energy resource supplied by the microgrid.

(iii) Static stochastic Nash games: A rather different motivation arises from considering a two-person stochastic Nash game of a rather special form. In such a game, the first agent is faced by a problem $\left(\mathrm{P}_{x}(\theta)\right)$ while the second agent faces a problem $\left(\mathrm{P}_{\theta}\right)$ which is independent of the first agent's decisions.

While we are unaware of the availability of general purpose mathematical tools that can resolve such problems, questions of a similar flavor have arisen in control-theoretic regimes. For instance, in adaptive control problems (Astrom and Wittenmark 1994), the true parameters may vary in time, and the control laws adapt to the changing parameters. Iterative learning control (Moore 1993) is a form of tracking control employed for repetitive control problems, instances being chemical batch processes, robot arm manipulators, and reliability testing rigs. Related questions also been studied in revenue management where Cooper, Homem-de Mello, and Kleywegt (2006) examined the devastating effect of learning with an incorrect model while maximizing revenue. Of note is also work on multi-armed bandit problems where resource allocation is considered in a problem whose parameters may be better understood with time (Gittins 1989). In related work by Jiang, Shanbhag, and Meyn (2011), the authors examine joint schemes for learning (misspecified) price function parameters and equilibrium computation in noise-corrupted Nash-Cournot games. In contrast with the current work, this approach accumulates observations while carrying out the computation. However, convergence guarantees are provided only in the mean-squared sense, rather than in a desirable almost-sure sense and no rate estimates are provided. In this paper, we develop coupled stochastic approximation schemes that produce sequences $\left\{x^{k}\right\}$ and $\left\{\theta^{k}\right\}$ such that

$$
\lim _{k \rightarrow \infty} x^{k}=x^{*} \text { and } \lim _{k \rightarrow \infty} \theta^{k}=\theta^{*}, \text { almost surely. }
$$

Additionally, rate estimates for such schemes are provided, both in a standard and in an averaging regime.

Before proceeding, it is worth commenting on why this problem is challenging. Under the assumption that $f(\bullet ; \theta)$ is convex in $(\bullet)$ for every $\theta \in \Theta$ and $g(\bullet)$ is convex in $(\bullet)$, the following holds:

$$
x^{*} \operatorname{solves}\left(P_{x}\left(\theta^{*}\right)\right) \text { and } \theta^{*} \text { solves }\left(P_{\theta}\right)
$$

if and only if $\left(x^{*}, \theta^{*}\right)$ is a solution to the (stochastic) variational inequality problem VI $(Z, F)$ where

$$
K \triangleq X \times \Theta \text { and } F(z, \theta) \triangleq\left(\begin{array}{c}
\nabla_{x} \mathbb{E}[f(x ; \theta, \xi)] \\
\nabla_{\theta}[g(\theta, \eta)]
\end{array}\right) .
$$

Recall that $z^{*}$ is a solution to VI $(K, F)$ if $\left(z-z^{*}\right)^{T} F(z) \geq 0$ for all $z \in K$. Furthermore, if $x^{*}$ and $\theta^{*}$ denote solutions to $\left(\mathrm{P}_{x}\left(\theta^{*}\right)\right.$ and $\left(\mathrm{P}_{\theta}\right)$, respectively, then an oft-used avenue in obtaining a solution $\left(x^{*}, \theta^{*}\right)$ entails obtaining a solution to $\mathrm{VI}(K, F)$. However, unless rather strong assumptions are imposed, the map $F$ is 
not necessarily monotone; recall that a map $F(z)$ is monotone over a set $K$ if $(F(x)-F(y))^{T}(x-y) \geq 0$ for all $x, y \in K$. Unfortunately, monotonicity of $F(z)$ is difficult to claim and this precludes the use of recently developed regularized stochastic approximation schemes for solving monotone stochastic variational inequality problems (Koshal, Nedic, and Shanbhag 2013).

The paper makes the following contributions: (i) In Section 2, we show that under suitable convexity and Lipschitz assumptions, our prescribed stochastic approximation schemes produce sequences that converge almost surely to a solution of the joint system. (ii) In Section 3, we develop rate estimates that rely on the usage of constant steplength schemes for the joint schemes in strongly convex and merely convex regimes, of which the latter leverages averaging techniques. The paper concludes with a brief set of remarks in Section 4. Finally, throughout the paper, we use $\|x\|$ to denote the Euclidean norm of a vector $x$, i.e., $\|x\|=\sqrt{x^{T} x}$ and $\Pi_{K}$ to denote the Euclidean projection operator onto a set $K$, i.e., $\Pi_{K}(x) \triangleq \operatorname{argmin}_{y \in K}\|x-y\|$.

\section{COUPLED STOCHASTIC APPROXIMATION SCHEMES}

As mentioned in the previous section, we propose a set of coupled stochastic approximation schemes for computing $x^{*}$ and $\theta^{*}$. Given $x^{0} \in X$ and $\theta^{0} \in \Theta$, the coupled SA schemes can be stated as follows:

Algorithm 1

$$
\begin{array}{rlrl}
x^{k+1} & :=\Pi_{X}\left(x^{k}-\gamma_{k, x}\left(\nabla_{x} f\left(x^{k} ; \theta^{k}\right)+w^{k}\right)\right), & & k \geq 0 \\
\theta^{k+1}:=\Pi_{\Theta}\left(\theta^{k}-\gamma_{k, \theta}\left(\nabla_{\theta} g\left(\theta^{k}\right)+v^{k}\right)\right), & & k \geq 0
\end{array}
$$

where $w^{k} \triangleq \nabla_{x} f\left(x^{k} ; \theta^{k}, \xi^{k}\right)-\nabla_{x} f\left(x^{k} ; \theta^{k}\right)$ and $v^{k} \triangleq \nabla_{\theta} g\left(\theta^{k} ; \eta^{k}\right)-\nabla_{\theta} g\left(\theta^{k}\right)$.

In this section, we develop the convergence theory for Algorithm 1 for settings where the function $f$ is both strongly convex and merely convex in $x$ for every $\theta \in \Theta$. We begin by stating an assumption on the functions $f$ and $g$.

Assumption 1 (A1) Suppose the following hold:

(i) For every $\theta \in \Theta, f(x ; \theta)$ is strongly convex and continuously differentiable with Lipschitz continuous gradients in $x$ with convexity constant $\mu_{x}$ and Lipschitz constant $L_{x}$, respectively.

(ii) For every $x \in X$, the gradient $\nabla_{x} f(x ; \theta)$ is Lipschitz continuous in $\theta$ with constant $L_{\theta}$.

(iii) The function $g(\theta)$ is strongly convex and continuously differentiable with Lipschitz continuous gradients in $\theta$ with convexity constant $\mu_{\theta}$ and Lipschitz constant $C_{\theta}$, respectively.

Additionally, we make the following assumptions on the steplength sequences employed in the algorithm.

Assumption 2 (A2) Let $\left\{\gamma_{k, x}\right\}$ and $\left\{\gamma_{k, \theta}\right\}$ be chosen such that:

$$
\begin{aligned}
& \sum_{k=0}^{\infty} \gamma_{k, x}=\infty, \sum_{k=0}^{\infty} \gamma_{k, x}^{2}<\infty . \\
& \gamma_{k, \theta}=\gamma_{k, x} L_{\theta}^{2} /\left(\mu_{x} \mu_{\theta}\right) .
\end{aligned}
$$

We define a new probability space $(Z, \mathscr{F}, \mathbb{P})$, where $Z \triangleq \Omega \times \Lambda, \mathscr{F} \triangleq \mathscr{F} x \times \mathscr{F} \theta$ and $\mathbb{P} \triangleq \mathbb{P}_{x} \times \mathbb{P}_{\theta}$. We use $\mathscr{F}_{k}$ to denote the sigma-field generated by the initial points $\left(x^{0}, \theta^{0}\right)$ and errors $\left(w^{l}, v^{l}\right)$ for $l=0,1, \cdots, k-1$, i.e., $\mathscr{F}_{0}=\left\{\left(x^{0}, \theta^{0}\right)\right\}$ and $\mathscr{F}_{k}=\left\{\left(x^{0}, \theta^{0}\right),\left(\left(w^{l}, v^{l}\right), l=0,1, \cdots, k-1\right)\right\}$ for $k \geq 1$. The following assumptions on the filtration and errors are made.

Assumption 3 (A3) Let the following hold:

(ii) $\quad \mathbb{E}\left[\left\|w^{k}\right\|^{2} \mid \mathscr{F}_{k}\right] \leq v_{x}^{2}$ and $\mathbb{E}\left[\left\|v^{k}\right\|^{2} \mid \mathscr{F}_{k}\right] \leq v_{\theta}^{2}$ a.s. for all $k$. 


\section{Jiang and Shanbhag}

We begin by providing a contraction result (without proof) which will be subsequently employed in developing our convergence statements.

Lemma 1 Let $H: K \rightarrow \mathbb{R}^{n}$ be a mapping that is strongly monotone over $K$ with constant $\mu$, and Lipschitz continuous over $K$ with constant $L$. If $q \triangleq \sqrt{1-2 \mu \gamma+\gamma^{2} L^{2}}$, then for any $\gamma>0$, we have the following:

$$
\left\|\Pi_{K}(x-\gamma H(x))-\Pi_{K}(y-\gamma H(y))\right\| \leq q\|x-y\|,
$$

In our analysis, we also use some well-known results on supermartingale convergence,

Lemma 2 (Polyak 1987, Lemma 10, Pg. 49) Let $V_{k}$ be a sequence of nonnegative random variables adapted to $\sigma$-algebra $\mathscr{F}_{k}$ and such that

$$
\mathbb{E}\left[V_{k+1} \mid \mathscr{F}_{k}\right] \leq\left(1-u_{k}\right) V_{k}+\beta_{k} \quad \text { for all } k \geq 0 \quad \text { almost surely, }
$$

where $0 \leq u_{k} \leq 1, \beta_{k} \geq 0$, and $\sum_{k=0}^{\infty} u_{k}=\infty, \sum_{k=0}^{\infty} \beta_{k}<\infty$ and $\lim _{k \rightarrow \infty} \frac{\beta_{k}}{u_{k}}=0$. Then, $V_{k} \rightarrow 0$ a.s.

Lemma 3 (Polyak 1987, Lemma 11, Pg. 50) Let $V_{k}, u_{k}, \beta_{k}$ and $\delta_{k}$ be nonnegative random variables adapted to $\sigma$-algebra $\mathscr{F}_{k}$. If $\sum_{k=0}^{\infty} u_{k}<\infty, \sum_{k=0}^{\infty} \beta_{k}<\infty$ and

$$
\mathbb{E}\left[V_{k+1} \mid \mathscr{F}_{k}\right] \leq\left(1+u_{k}\right) V_{k}-\delta_{k}+\beta_{k} \quad \text { for all } k \geq 0 \quad \text { almost surely. }
$$

Then, $\left\{V_{k}\right\}$ is convergent and $\sum_{k=0}^{\infty} \delta_{k}<\infty$ almost surely.

We now leverage these results in examining the convergence properties of Algorithm 1.

Theorem 4 (Almost-sure convergence under strong convexity of $f$ ) Suppose (A1), (A2) and (A3) hold. Let $\left\{x^{k}, \theta^{k}\right\}$ be computed via Algorithm 1. Then, $x^{k} \rightarrow x^{*}$ and $\theta^{k} \rightarrow \theta^{*}$ a.s. as $k \rightarrow \infty$, where $x^{*}$ is the unique solution to $\left(\mathrm{P}_{x}\left(\theta^{*}\right)\right)$.

Proof: Note that $x^{*}=\Pi_{X}\left(x^{*}-\gamma_{k, x} \nabla_{x} f\left(x^{*} ; \theta^{*}\right)\right)$. Then, by the nonexpansivity of the Euclidean projector, $\left\|x^{k+1}-x^{*}\right\|^{2}$ may be bounded as follows:

$$
\begin{aligned}
\left\|x^{k+1}-x^{*}\right\|^{2} & =\left\|\Pi_{X}\left(x^{k}-\gamma_{k, x}\left(\nabla_{x} f\left(x^{k} ; \theta^{k}\right)+w^{k}\right)\right)-\Pi_{X}\left(x^{*}-\gamma_{k, x} \nabla_{x} f\left(x^{*} ; \theta^{*}\right)\right)\right\|^{2} \\
& \leq\left\|\left(x^{k}-x^{*}\right)-\gamma_{k, x}\left(\nabla_{x} f\left(x^{k} ; \theta^{k}\right)-\nabla_{x} f\left(x^{*} ; \theta^{*}\right)\right)-\gamma_{k, x} w^{k}\right\|^{2} .
\end{aligned}
$$

By adding and subtracting $\gamma_{k, x} \nabla_{x} f\left(x^{*}, \theta^{k}\right)$, this expression can be further expanded as follows:

$$
\begin{aligned}
& \left\|\left(x^{k}-x^{*}\right)-\gamma_{k, x}\left(\nabla_{x} f\left(x^{k} ; \theta^{k}\right)-\nabla_{x} f\left(x^{*} ; \theta^{k}\right)\right)-\gamma_{k, x}\left(\nabla_{x} f\left(x^{*} ; \theta^{k}\right)-\nabla_{x} f\left(x^{*} ; \theta^{*}\right)\right)-\gamma_{k, x} w^{k}\right\|^{2} \\
= & \left\|\left(x^{k}-x^{*}\right)-\gamma_{k, x}\left(\nabla_{x} f\left(x^{k} ; \theta^{k}\right)-\nabla_{x} f\left(x^{*} ; \theta^{k}\right)\right)\right\|^{2}+\gamma_{k, x}^{2}\left\|\nabla_{x} f\left(x^{*} ; \theta^{k}\right)-\nabla_{x} f\left(x^{*} ; \theta^{*}\right)\right\|^{2}+\gamma_{k, x}^{2}\left\|w^{k}\right\|^{2} \\
& -2 \gamma_{k, x}\left[\left(x^{k}-x^{*}\right)-\gamma_{k, x}\left(\nabla_{x} f\left(x^{k} ; \theta^{k}\right)-\nabla_{x} f\left(x^{*} ; \theta^{k}\right)\right)\right]^{T}\left(\nabla_{x} f\left(x^{*} ; \theta^{k}\right)-\nabla_{x} f\left(x^{*} ; \theta^{*}\right)\right) \\
& -2 \gamma_{k, x}\left[\left(x^{k}-x^{*}\right)-\gamma_{k, x}\left(\nabla_{x} f\left(x^{k} ; \theta^{k}\right)-\nabla_{x} f\left(x^{*} ; \theta^{k}\right)\right)\right]^{T} w^{k} \\
& +2 \gamma_{k, x}^{2}\left(\nabla_{x} f\left(x^{*} ; \theta^{k}\right)-\nabla_{x} f\left(x^{*} ; \theta^{*}\right)\right)^{T} w^{k} .
\end{aligned}
$$

By leveraging the fact that $\mathbb{E}\left[w^{k} \mid \mathscr{F}_{k}\right]=0$ and $\mathbb{E}\left[\left\|w^{k}\right\|^{2} \mid \mathscr{F}_{k}\right] \leq v_{x}^{2}$, we have

$$
\mathbb{E}\left[\left\|x^{k+1}-x^{*}\right\|^{2} \mid \mathscr{F}_{k}\right] \leq \operatorname{Term} 1+\operatorname{Term} 2+\operatorname{Term} 3+\gamma_{k, x}^{2} v_{x}^{2},
$$

where Terms $\mathbf{1}-\mathbf{3}$ are defined as follows:

Term $\mathbf{1} \triangleq\left\|\left(x^{k}-x^{*}\right)-\gamma_{k, x}\left(\nabla_{x} f\left(x^{k} ; \theta^{k}\right)-\nabla_{x} f\left(x^{*} ; \theta^{k}\right)\right)\right\|^{2}$, Term $\mathbf{2} \triangleq \gamma_{k, x}^{2}\left\|\nabla_{x} f\left(x^{*} ; \theta^{k}\right)-\nabla_{x} f\left(x^{*} ; \theta^{*}\right)\right\|^{2}$, and Term $3 \triangleq-2 \gamma_{k, x}\left[\left(x^{k}-x^{*}\right)-\gamma_{k, x}\left(\nabla_{x} f\left(x^{k} ; \theta^{k}\right)-\nabla_{x} f\left(x^{*} ; \theta^{k}\right)\right)\right]^{T}\left(\nabla_{x} f\left(x^{*} ; \theta^{k}\right)-\nabla_{x} f\left(x^{*} ; \theta^{*}\right)\right)$. 
By Lemma 1 and (A1), it follows that

$$
\text { Term } 1 \leq\left(1-2 \gamma_{k, x} \mu_{x}+\gamma_{k, x}^{2} L_{x}^{2}\right)\left\|x^{k}-x^{*}\right\|^{2} .
$$

Furthermore, the Lipschitz continuity of $\nabla_{x} f\left(x^{*} ; \theta\right)$ in $\theta$ (A1) allows for deriving the following bound:

$$
\text { Term } 2 \leq \gamma_{k, x}^{2} L_{\theta}^{2}\left\|\theta^{k}-\theta^{*}\right\|^{2}
$$

Finally, Term 3 can be bounded by invoking the Cauchy-Schwarz inequality, Lemma 1, (A1) and the triangle inequality, we obtain

$$
\begin{aligned}
& 2 \gamma_{k, x}\left\|\left(x^{k}-x^{*}\right)-\gamma_{k, x}\left(\nabla_{x} f\left(x^{k} ; \theta^{k}\right)-\nabla_{x} f\left(x^{*} ; \theta^{k}\right)\right)\right\|\left\|\nabla_{x} f\left(x^{*} ; \theta^{k}\right)-\nabla_{x} f\left(x^{*} ; \theta^{*}\right)\right\| \\
\leq & 2 \gamma_{k, x} \sqrt{1-2 \gamma_{k, x} \mu_{x}+\gamma_{k, x}^{2} L_{x}^{2}}\left\|x^{k}-x^{*}\right\| \cdot L_{\theta}\left\|\theta^{k}-\theta^{*}\right\| \leq 2 \gamma_{k, x} L_{\theta}\left\|x^{k}-x^{*}\right\|\left\|\theta^{k}-\theta^{*}\right\| \\
\leq & \gamma_{k, x} \mu_{x}\left\|x^{k}-x^{*}\right\|^{2}+\gamma_{k, x}\left(L_{\theta}^{2} / \mu_{x}\right)\left\|\theta^{k}-\theta^{*}\right\|^{2} .
\end{aligned}
$$

The first inequality follows from the strong monotonicity and Lipschitz continuity of $\nabla_{x} f(x ; \theta)$ in $x$ and Lemma 1 , and the Lipschitz continuity of $\nabla_{x} f(x ; \theta)$ in $\theta$. The last inequality follows from $2 a^{T} b \leq$ $\|a\|^{2}+\|b\|^{2}$. Combining (3), (4), (5) and (6), we get

$$
\mathbb{E}\left[\left\|x^{k+1}-x^{*}\right\|^{2} \mid \mathscr{F}_{k}\right] \leq\left(1-\gamma_{k, x} \mu_{x}+\gamma_{k, x}^{2} L_{x}^{2}\right)\left\|x^{k}-x^{*}\right\|^{2}+\left(\gamma_{k, x} L_{\theta}^{2} / \mu_{x}+\gamma_{k, x}^{2} L_{\theta}^{2}\right)\left\|\theta^{k}-\theta^{*}\right\|^{2}+\gamma_{k, x}^{2} v_{x}^{2} .
$$

Recall that $\theta^{*}$ satisfies the fixed point relationship $\theta^{*}=\Pi_{\Theta}\left(\theta^{*}-\gamma_{\theta, k} \nabla_{\theta} g_{\theta}\left(\theta^{*}\right)\right)$, which, together with non-expansivity of the Euclidean projector, allows for deriving the following bound on $\left\|\theta^{k+1}-\theta^{*}\right\|^{2}$ :

$$
\begin{aligned}
\left\|\theta^{k+1}-\theta^{*}\right\|^{2}= & \left\|\Pi_{\Theta}\left(\theta^{k}-\gamma_{\theta, k}\left(\nabla_{\theta} g\left(\theta^{k}\right)+v^{k}\right)\right)-\Pi_{\Theta}\left(\theta^{*}-\gamma_{\theta, k} \nabla_{\theta} g_{\theta}\left(\theta^{*}\right)\right)\right\|^{2} \\
\leq & \left\|\theta^{k}-\theta^{*}-\gamma_{\theta, k}\left(\nabla_{\theta} g_{\theta}\left(\theta^{k}\right)-\nabla_{\theta} g_{\theta}\left(\theta^{*}\right)\right)-\gamma_{\theta, k} v^{k}\right\|^{2} \\
= & \left\|\theta^{k}-\theta^{*}-\gamma_{\theta, k}\left(\nabla_{\theta} g_{\theta}\left(\theta^{k}\right)-\nabla_{\theta} g_{\theta}\left(\theta^{*}\right)\right)\right\|^{2}+\gamma_{\theta, k}^{2}\left\|v^{k}\right\|^{2} \\
& -2\left(\theta^{k}-\theta^{*}-\gamma_{\theta, k}\left(\nabla_{\theta} g_{\theta}\left(\theta^{k}\right)-\nabla_{\theta} g_{\theta}\left(\theta^{*}\right)\right)\right)^{T} v^{k} .
\end{aligned}
$$

By taking conditional expectations and by recalling that $\mathbb{E}\left[v^{k} \mid \mathscr{F}_{k}\right]=0$ and $\mathbb{E}\left[\left\|v^{k}\right\|^{2} \mid \mathscr{F}_{k}\right] \leq v_{\theta}^{2}$, we obtain the following:

$$
\begin{aligned}
\mathbb{E}\left[\left\|\theta^{k+1}-\theta^{*}\right\|^{2} \mid \mathscr{F}_{k}\right] & \leq\left\|\theta^{k}-\theta^{*}-\gamma_{k, \theta}\left(\nabla_{\theta} g_{\theta}\left(\theta^{k}\right)-\nabla_{\theta} g_{\theta}\left(\theta^{*}\right)\right)\right\|^{2}+\gamma_{k, \theta}^{2} \mathbb{E}\left[\left\|v^{k}\right\|^{2} \mid \mathscr{F}_{k}\right] \\
& \leq q_{k, \theta}^{2}\left\|\theta^{k}-\theta^{*}\right\|^{2}+\gamma_{k, \theta}^{2} v_{\theta}^{2}
\end{aligned}
$$

where $q_{k, \theta} \triangleq \sqrt{1-2 \gamma_{k, \theta} \mu_{\theta}+\gamma_{k, \theta}^{2} C_{\theta}^{2}}$. Next, by adding (7) and (9) and using the assumption $\gamma_{k, \theta}=$ $\gamma_{k, x} L_{\theta}^{2} /\left(\mu_{x} \mu_{\theta}\right)$ (A2 (ii)), we obtain the following bound.

$$
\begin{aligned}
\mathbb{E}\left[\left\|x^{k+1}-x^{*}\right\|^{2} \mid \mathscr{F}_{k}\right]+ & \mathbb{E}\left[\left\|\theta^{k+1}-\theta^{*}\right\|^{2} \mid \mathscr{F}_{k}\right] \leq\left(1-\gamma_{k, x} \mu_{x}+\gamma_{k, x}^{2} L_{x}^{2}\right)\left\|x^{k}-x^{*}\right\|^{2} \\
& +\left(q_{k, \theta}^{2}+\gamma_{k, x} L_{\theta}^{2} / \mu_{x}+\gamma_{k, x}^{2} L_{\theta}^{2}\right)\left\|\theta^{k}-\theta^{*}\right\|^{2}+\gamma_{k, x}^{2} v_{x}^{2}+\gamma_{k, \theta}^{2} v_{\theta}^{2} \\
= & \left(1-\gamma_{k, x} \mu_{x}+\gamma_{k, x}^{2} L_{x}^{2}\right)\left\|x^{k}-x^{*}\right\|^{2}+\left(1-\gamma_{k, x} L_{\theta}^{2} / \mu_{x}+\gamma_{k, x}^{2}\left(L_{\theta}^{2}+L_{\theta}^{4} C_{\theta}^{2} /\left(\mu_{x}^{2} \mu_{\theta}^{2}\right)\right)\right)\left\|\theta^{k}-\theta^{*}\right\|^{2} \\
& +\gamma_{k, x}^{2} v_{x}^{2}+\gamma_{k, x}^{2} v_{\theta}^{2} L_{\theta}^{4} /\left(\mu_{x}^{2} \mu_{\theta}^{2}\right) \\
\leq & \left(1-\alpha \gamma_{k, x}+\beta \gamma_{k, x}^{2}\right)\left(\left\|x^{k}-x^{*}\right\|^{2}+\left\|\theta^{k}-\theta^{*}\right\|^{2}\right)+\delta \gamma_{k, x}^{2}
\end{aligned}
$$

where $\alpha=\min \left\{\mu_{x}, L_{\theta}^{2} / \mu_{x}\right\}, \beta=\max \left\{L_{x}^{2}, L_{\theta}^{2}+L_{\theta}^{4} C_{\theta}^{2} /\left(\mu_{x}^{2} \mu_{\theta}^{2}\right)\right\}$ and $\delta=v_{x}^{2}+v_{\theta}^{2} L_{\theta}^{4} /\left(\mu_{x}^{2} \mu_{\theta}^{2}\right)$. From (A2), we have that $\sum_{k=0}^{\infty}\left(\alpha \gamma_{k, x}-\beta \gamma_{k, x}^{2}\right)=\infty, \quad \sum_{k=0}^{\infty} \delta \gamma_{k, x}^{2}<\infty$ and $\lim _{k \rightarrow \infty} \frac{\delta \gamma_{k, x}^{2}}{\alpha \gamma_{k, x}-\beta \gamma_{k, x}^{2}}=0$. Then, by invoking the super-martingale convergence theorem (Lemma 2), we have that $\left\|x^{k}-x^{*}\right\|^{2}+\left\|\theta^{k}-\theta^{*}\right\|^{2} \rightarrow 0$ a.s. as $k \rightarrow \infty$, which implies that $x^{k} \rightarrow x^{*}$ and $\theta^{k} \rightarrow \theta^{*}$ a.s. as $k \rightarrow \infty$.

Next we weaken the strong convexity requirement on the function $f$ through the following assumption. 


\section{Jiang and Shanbhag}

Assumption 4 (A4) Suppose the following holds in addition to (A1 (ii)) and (A1 (iii)).

(i) For every $\theta \in \Theta, f(x ; \theta)$ is convex and continuously differentiable with Lipschitz continuous gradients in $x$ with Lipschitz constant $L_{x}$.

We make the following assumptions on the steplength sequences employed in the algorithm.

Assumption 5 (A5) Let $\left\{\gamma_{k, x}\right\},\left\{\gamma_{k, \theta}\right\}$ and some constant $\tau \in(0,1)$ be chosen such that:

$$
\begin{aligned}
& \sum_{k=0}^{\infty} \gamma_{k, x}^{2-\tau}<\infty \text { and } \sum_{k=0}^{\infty} \gamma_{k, \theta}^{2}<\infty, \\
& \sum_{k=0}^{\infty} \gamma_{k, x}=\infty \text { and } \sum_{k=0}^{\infty} \gamma_{k, \theta}=\infty, \\
& \beta_{k}=\frac{\gamma_{k, x}}{2 \gamma_{k, \theta} \mu_{\theta}} \downarrow 0 \text { as } k \rightarrow \infty .
\end{aligned}
$$

The convergence result for Algorithm 1 can be stated as follows.

Theorem 5 (Almost-sure convergence under convexity of $f$ ) Suppose (A3), (A4) and (A5) hold. Suppose $X$ is bounded and the solution set $X^{*}$ of $\left(\mathrm{P}_{x}\left(\theta^{*}\right)\right)$ is nonempty. Let $\left\{x^{k}, \theta^{k}\right\}$ be computed via Algorithm 1. Then, $\theta^{k} \rightarrow \theta^{*}$ a.s. as $k \rightarrow \infty$, and $x^{k}$ converges to a random point in $X^{*}$ a.s. as $k \rightarrow \infty$.

Proof: By the nonexpansivity of the Euclidean projector, we have for any $x^{*} \in X^{*}$ that

$$
\left\|x^{k+1}-x^{*}\right\|^{2}=\left\|\Pi_{X}\left(x^{k}-\gamma_{k, x}\left(\nabla_{x} f\left(x^{k} ; \theta^{k}\right)+w^{k}\right)\right)-\Pi_{X}\left(x^{*}\right)\right\|^{2} \leq\left\|\left(x^{k}-x^{*}\right)-\gamma_{k, x} \nabla_{x} f\left(x^{k} ; \theta^{k}\right)-\gamma_{k, x} w^{k}\right\|^{2} .
$$

By adding and subtracting $\gamma_{k, x} \nabla_{x} f\left(x^{*}, \theta^{k}\right)$, this expression can be further expanded as follows:

$$
\begin{aligned}
& \left\|\left(x^{k}-x^{*}\right)-\gamma_{k, x} \nabla_{x} f\left(x^{k} ; \theta^{*}\right)-\gamma_{k, x}\left(\nabla_{x} f\left(x^{k} ; \theta^{k}\right)-\nabla_{x} f\left(x^{k} ; \theta^{*}\right)\right)-\gamma_{k, x} w^{k}\right\|^{2} \\
= & \left\|\left(x^{k}-x^{*}\right)-\gamma_{k, x} \nabla_{x} f\left(x^{k} ; \theta^{*}\right)\right\|^{2}+\gamma_{k, x}^{2}\left\|\nabla_{x} f\left(x^{k} ; \theta^{k}\right)-\nabla_{x} f\left(x^{k} ; \theta^{*}\right)\right\|^{2}+\gamma_{k, x}^{2}\left\|w^{k}\right\|^{2} \\
& -2 \gamma_{k, x}\left[\left(x^{k}-x^{*}\right)-\gamma_{k, x} \nabla_{x} f\left(x^{k} ; \theta^{*}\right)\right]^{T}\left(\nabla_{x} f\left(x^{k} ; \theta^{k}\right)-\nabla_{x} f\left(x^{k} ; \theta^{*}\right)\right) \\
& -2 \gamma_{k, x}\left[\left(x^{k}-x^{*}\right)-\gamma_{k, x} \nabla_{x} f\left(x^{k} ; \theta^{*}\right)\right]^{T} w^{k}+2 \gamma_{k, x}^{2}\left(\nabla_{x} f\left(x^{k} ; \theta^{k}\right)-\nabla_{x} f\left(x^{k} ; \theta^{*}\right)\right)^{T} w^{k} .
\end{aligned}
$$

Noting that $\mathbb{E}\left[w^{k} \mid \mathscr{F}_{k}\right]=0$ and $\mathbb{E}\left[\left\|w^{k}\right\|^{2} \mid \mathscr{F}_{k}\right] \leq v_{x}^{2}$, we have

$$
\mathbb{E}\left[\left\|x^{k+1}-x^{*}\right\|^{2} \mid \mathscr{F}_{k}\right] \leq \operatorname{Term} 1+\operatorname{Term} 2+\operatorname{Term} 3+\gamma_{k, x}^{2} v_{x}^{2},
$$

where Terms $\mathbf{1}-\mathbf{3}$ are defined as follows:

Term $1 \triangleq\left\|\left(x^{k}-x^{*}\right)-\gamma_{k, x} \nabla_{x} f\left(x^{k} ; \theta^{*}\right)\right\|^{2}$, Term $2 \triangleq \gamma_{k, x}^{2}\left\|\nabla_{x} f\left(x^{k} ; \theta^{k}\right)-\nabla_{x} f\left(x^{k} ; \theta^{*}\right)\right\|^{2}$, and Term $3 \triangleq-2 \gamma_{k, x}\left[\left(x^{k}-x^{*}\right)-\gamma_{k, x} \nabla_{x} f\left(x^{k} ; \theta^{*}\right)\right]^{T}\left(\nabla_{x} f\left(x^{k} ; \theta^{k}\right)-\nabla_{x} f\left(x^{k} ; \theta^{*}\right)\right)$.

By invoking the convexity of $f(x ; \theta)$ in $x$ and the gradient inequality (see A4), we have that

$$
\text { Term } \begin{aligned}
1 & =\left\|x^{k}-x^{*}\right\|^{2}+\gamma_{k, x}^{2}\left\|\nabla_{x} f\left(x^{k} ; \theta^{*}\right)\right\|^{2}-2 \gamma_{k, x}\left(x^{k}-x^{*}\right)^{T} \nabla_{x} f\left(x^{k} ; \theta^{*}\right) \\
& \leq\left\|x^{k}-x^{*}\right\|^{2}+\gamma_{k, x}^{2}\left\|\nabla_{x} f\left(x^{k} ; \theta^{*}\right)\right\|^{2}-2 \gamma_{k, x}\left(f\left(x^{k} ; \theta^{*}\right)-f\left(x^{*} ; \theta^{*}\right)\right) \\
& \leq\left\|x^{k}-x^{*}\right\|^{2}+2 \gamma_{k, x}^{2}\left\|\nabla_{x} f\left(x^{k} ; \theta^{*}\right)-\nabla_{x} f\left(x^{*} ; \theta^{*}\right)\right\|^{2}+2 \gamma_{k, x}^{2}\left\|\nabla_{x} f\left(x^{*} ; \theta^{*}\right)\right\|^{2}-2 \gamma_{k, x}\left(f\left(x^{k} ; \theta^{*}\right)-f\left(x^{*} ; \theta^{*}\right)\right),
\end{aligned}
$$

where the last inequality follows from the identity $\|(a-b)+b\|^{2} \leq 2\|a-b\|^{2}+2\|b\|^{2}$. From the Lipschitz continuity of $\nabla_{x} f(x ; \theta)$ in $x$, the right hand side can be bounded as follows:

$$
\begin{aligned}
& \left\|x^{k}-x^{*}\right\|^{2}+2 \gamma_{k, x}^{2}\left\|\nabla_{x} f\left(x^{k} ; \theta^{*}\right)-\nabla_{x} f\left(x^{*} ; \theta^{*}\right)\right\|^{2}+2 \gamma_{k, x}^{2}\left\|\nabla_{x} f\left(x^{*} ; \theta^{*}\right)\right\|^{2}-2 \gamma_{k, x}\left(f\left(x^{k} ; \theta^{*}\right)-f\left(x^{*} ; \theta^{*}\right)\right) \\
\leq & \left(1+2 \gamma_{k, x}^{2} L_{x}^{2}\right)\left\|x^{k}-x^{*}\right\|^{2}+2 \gamma_{k, x}^{2}\left\|\nabla_{x} f\left(x^{*} ; \theta^{*}\right)\right\|^{2}-2 \gamma_{k, x}\left(f\left(x^{k} ; \theta^{*}\right)-f\left(x^{*} ; \theta^{*}\right)\right) .
\end{aligned}
$$




\section{Jiang and Shanbhag}

By the Lipschitz continuity of $\nabla_{x} f(x ; \theta)$ in $\theta$ (A4),

$$
\operatorname{Term} 2 \leq \gamma_{k, x}^{2} L_{\theta}^{2}\left\|\theta^{k}-\theta^{*}\right\|^{2} .
$$

By adding and subtracting $\nabla_{x} f\left(x^{*} ; \theta^{*}\right)$, and by invoking the Lipschitz continuity of $\nabla_{x} f(x ; \theta)$ in $x$, (A4) and the triangle inequality, we may derive a bound for Term $\mathbf{3}$ as follows:

$$
\text { Term } \begin{aligned}
3 & \leq 2 \gamma_{k, x}\left\|\left(x^{k}-x^{*}\right)-\gamma_{k, x} \nabla_{x} f\left(x^{k} ; \theta^{*}\right)\right\|\left\|\nabla_{x} f\left(x^{k} ; \theta^{k}\right)-\nabla_{x} f\left(x^{k} ; \theta^{*}\right)\right\| \\
& \leq 2 \gamma_{k, x}\left\|\left(x^{k}-x^{*}\right)-\gamma_{k, x}\left(\nabla_{x} f\left(x^{k} ; \theta^{*}\right)-\nabla_{x} f\left(x^{*} ; \theta^{*}\right)\right)-\gamma_{k, x} \nabla_{x} f\left(x^{*} ; \theta^{*}\right)\right\| \cdot L_{\theta}\left\|\theta^{k}-\theta^{*}\right\| \\
& \leq 2 \gamma_{k, x}\left(\left(1+\gamma_{k, x} L_{x}\right)\left\|x^{k}-x^{*}\right\|+\gamma_{k, x}\left\|\nabla_{x} f\left(x^{*} ; \theta^{*}\right)\right\|\right) L_{\theta}\left\|\theta^{k}-\theta^{*}\right\| \\
& =2 \gamma_{k, x} L_{\theta}\left\|x^{k}-x^{*}\right\|\left\|\theta^{k}-\theta^{*}\right\|+2 \gamma_{k, x}^{2} L_{\theta} L_{x}\left\|x^{k}-x^{*}\right\|\left\|\theta^{k}-\theta^{*}\right\|+2 \gamma_{k, x}^{2} L_{\theta}\left\|\nabla_{x} f\left(x^{*} ; \theta^{*}\right)\right\|\left\|\theta^{k}-\theta^{*}\right\| .
\end{aligned}
$$

By using the fact that $2 a b \leq a^{2}+b^{2}$, we further have the following:

$$
\begin{aligned}
\operatorname{Term} 3 \leq & \gamma_{k, x}^{2-\tau} L_{\theta}^{2}\left\|x^{k}-x^{*}\right\|^{2}+\gamma_{k, x}^{\tau}\left\|\theta^{k}-\theta^{*}\right\|^{2}+\gamma_{k, x}^{2} L_{\theta} L_{x}\left\|x^{k}-x^{*}\right\|^{2}+\gamma_{k, x}^{2} L_{\theta} L_{x}\left\|\theta^{k}-\theta^{*}\right\|^{2} \\
& +\gamma_{k, x}^{2} L_{\theta}^{2}\left\|\theta^{k}-\theta^{*}\right\|^{2}+\gamma_{k, x}^{2}\left\|\nabla_{x} f\left(x^{*} ; \theta^{*}\right)\right\|^{2}
\end{aligned}
$$

where $\tau \in(0,1)$ is chosen to satisfy (A5). Combining (12), (13), (14) and (16), we obtain the following bound on the conditional error.

$$
\begin{aligned}
\mathbb{E}\left[\left\|x^{k+1}-x^{*}\right\|^{2} \mid \mathscr{F}_{k}\right] \leq & \left(1+\gamma_{k, x}^{2-\tau} L_{\theta}^{2}+\gamma_{k, x}^{2}\left(2 L_{x}^{2}+L_{\theta} L_{x}\right)\right)\left\|x^{k}-x^{*}\right\|^{2}+\left(\gamma_{k, x}^{\tau}+\gamma_{k, x}^{2}\left(2 L_{\theta}^{2}+L_{\theta} L_{x}\right)\right)\left\|\theta^{k}-\theta^{*}\right\|^{2} \\
& +3 \gamma_{k, x}^{2}\left\|\nabla_{x} f\left(x^{*} ; \theta^{*}\right)\right\|^{2}-2 \gamma_{k, x}\left(f\left(x^{k} ; \theta^{*}\right)-f\left(x^{*} ; \theta^{*}\right)\right)+\gamma_{k, x}^{2} v_{x}^{2} .
\end{aligned}
$$

From (9), we have that

$$
\mathbb{E}\left[\left\|\theta^{k+1}-\theta^{*}\right\|^{2} \mid \mathscr{F}_{k}\right] \leq q_{k, \theta}^{2}\left\|\theta^{k}-\theta^{*}\right\|^{2}+\gamma_{k, \theta}^{2} v_{\theta}^{2},
$$

where $q_{k, \theta} \triangleq \sqrt{1-2 \gamma_{k, \theta} \mu_{\theta}+\gamma_{k, \theta}^{2} C_{\theta}^{2}}$. Choose $\beta_{k}=\frac{\gamma_{k, x}^{\tau}}{2 \gamma_{k, \theta} \mu_{\theta}}$ by (A5). Note that by assumption $\beta_{k+1} \leq \beta_{k}$. By multiplying the left hand side of (18) by $\beta_{k+1}$ and adding to the left hand side of (17), we get

$$
\begin{aligned}
& \mathbb{E}\left[\left\|x^{k+1}-y^{k}\right\|^{2} \mid \mathscr{F}_{k}\right]+\beta_{k+1} \mathbb{E}\left[\left\|\theta^{k+1}-\theta^{*}\right\|^{2} \mid \mathscr{F}_{k}\right] \leq \mathbb{E}\left[\left\|x^{k+1}-y^{k}\right\|^{2} \mid \mathscr{F}_{k}\right]+\beta_{k} \mathbb{E}\left[\left\|\theta^{k+1}-\theta^{*}\right\|^{2} \mid \mathscr{F}_{k}\right] \\
\leq & \left(1+\gamma_{k, x}^{2-\tau} L_{\theta}^{2}+\gamma_{k, x}^{2}\left(2 L_{x}^{2}+L_{\theta} L_{x}\right)\right)\left\|x^{k}-x^{*}\right\|^{2}+\left(\beta_{k} q_{k, \theta}^{2}+\gamma_{k, x}^{\tau}+\gamma_{k, x}^{2}\left(2 L_{\theta}^{2}+L_{\theta} L_{x}\right)\right)\left\|\theta^{k}-\theta^{*}\right\|^{2} \\
& +3 \gamma_{k, x}^{2}\left\|\nabla_{x} f\left(x^{*} ; \theta^{*}\right)\right\|^{2}+\beta_{k} \gamma_{k, \theta}^{2} v_{\theta}^{2}+\gamma_{k, x}^{2} v_{x}^{2}-2 \gamma_{k, x}\left(f\left(x^{k} ; \theta^{*}\right)-f\left(x^{*} ; \theta^{*}\right)\right) \\
\leq & \left(1+\gamma_{k, x}^{2-\tau} L_{\theta}^{2}+\gamma_{k, x}^{2}\left(2 L_{x}^{2}+L_{\theta} L_{x}\right)\right)\left\|x^{k}-x^{*}\right\|^{2}+\underbrace{\frac{\beta_{k} q_{k, \theta}^{2}+\gamma_{k, x}^{\tau}+\gamma_{k, x}^{2}\left(2 L_{\theta}^{2}+L_{\theta} L_{x}\right)}{\beta_{k}}}_{\text {Term } 4} \cdot \beta_{k}\left\|\theta^{k}-\theta^{*}\right\|^{2} \\
& +3 \gamma_{k, x}^{2}\left\|\nabla_{x} f\left(x^{*} ; \theta^{*}\right)\right\|^{2}+\beta_{k} \gamma_{k, \theta}^{2} v_{\theta}^{2}+\gamma_{k, x}^{2} v_{x}^{2}-2 \gamma_{k, x}\left(f\left(x^{k} ; \theta^{*}\right)-f\left(x^{*} ; \theta^{*}\right)\right) .
\end{aligned}
$$

Term 4 on the right hand side of (19) can be further expanded as

$$
\begin{aligned}
& \frac{\beta_{k} q_{k, \theta}^{2}+\gamma_{k, x}^{\tau}+\gamma_{k, x}^{2}\left(2 L_{\theta}^{2}+L_{\theta} L_{x}\right)}{\beta_{k}}=q_{k, \theta}^{2}+\frac{\gamma_{k, x}^{\tau}+\gamma_{k, x}^{2}\left(2 L_{\theta}^{2}+L_{\theta} L_{x}\right)}{\beta_{k}} \\
& =1-2 \gamma_{k, \theta} \mu_{\theta}+\gamma_{k, \theta}^{2} C_{\theta}^{2}+\frac{\gamma_{k, x}^{\tau}}{\beta_{k}}+\frac{\gamma_{k, x}^{2}\left(2 L_{\theta}^{2}+L_{\theta} L_{x}\right)}{\beta_{k}}=1+\gamma_{k, \theta}^{2} C_{\theta}^{2}+2 \gamma_{k, \theta} \gamma_{k, x}^{2-\tau} \mu_{\theta}\left(2 L_{\theta}^{2}+L_{\theta} L_{x}\right) .
\end{aligned}
$$


Combining (19) and (20), we get

$$
\begin{aligned}
\mathbb{E} & {\left[\left\|x^{k+1}-y^{k}\right\|^{2} \mid \mathscr{F}_{k}\right]+\beta_{k+1} \mathbb{E}\left[\left\|\theta^{k+1}-\theta^{*}\right\|^{2} \mid \mathscr{F}_{k}\right] \leq\left(1+\gamma_{k, x}^{2-\tau} L_{\theta}^{2}+\gamma_{k, x}^{2}\left(2 L_{x}^{2}+L_{\theta} L_{x}\right)\right)\left\|x^{k}-x^{*}\right\|^{2} } \\
& +\left(1+\gamma_{k, \theta}^{2} C_{\theta}^{2}+2 \gamma_{k, \theta} \gamma_{k, x}^{2-\tau} \mu_{\theta}\left(2 L_{\theta}^{2}+L_{\theta} L_{x}\right)\right) \beta_{k}\left\|\theta^{k}-\theta^{*}\right\|^{2} \\
& +3 \gamma_{k, x}^{2}\left\|\nabla_{x} f\left(x^{*} ; \theta^{*}\right)\right\|^{2}+\beta_{k} \gamma_{k, \theta}^{2} v_{\theta}^{2}+\gamma_{k, x}^{2} v_{x}^{2}-2 \gamma_{k, x}\left(f\left(x^{k} ; \theta^{*}\right)-f\left(x^{*} ; \theta^{*}\right)\right) \\
\leq & \left(1+\gamma_{k, \theta}^{2} C_{\theta}^{2}+2 \gamma_{k, \theta} \gamma_{k, x}^{2-\tau} \mu_{\theta}\left(2 L_{\theta}^{2}+L_{\theta} L_{x}\right)\right)\left(\left\|x^{k}-x^{*}\right\|^{2}+\beta_{k}\left\|\theta^{k}-\theta^{*}\right\|^{2}\right) \\
& +\left(\gamma_{k, x}^{2-\tau} L_{\theta}^{2}+\gamma_{k, x}^{2}\left(2 L_{x}^{2}+L_{\theta} L_{x}\right)-\gamma_{k, \theta}^{2} C_{\theta}^{2}-2 \gamma_{k, \theta} \gamma_{k, x}^{2-\tau} \mu_{\theta}\left(2 L_{\theta}^{2}+L_{\theta} L_{x}\right)\right)\left\|x^{k}-x^{*}\right\|^{2} \\
& +3 \gamma_{k, x}^{2}\left\|\nabla_{x} f\left(x^{*} ; \theta^{*}\right)\right\|^{2}+\beta_{k} \gamma_{k, \theta}^{2} v_{\theta}^{2}+\gamma_{k, x}^{2} v_{x}^{2}-2 \gamma_{k, x}\left(f\left(x^{k} ; \theta^{*}\right)-f\left(x^{*} ; \theta^{*}\right)\right) .
\end{aligned}
$$

Let $u_{k} \triangleq \gamma_{k, \theta}^{2} C_{\theta}^{2}+2 \gamma_{k, \theta} \gamma_{k, x}^{2-\tau} \mu_{\theta}\left(2 L_{\theta}^{2}+L_{\theta} L_{x}\right)$,

$$
\begin{aligned}
\rho_{k} \triangleq & \left(\gamma_{k, x}^{2-\tau} L_{\theta}^{2}+\gamma_{k, x}^{2}\left(2 L_{x}^{2}+L_{\theta} L_{x}\right)-\gamma_{k, \theta}^{2} C_{\theta}^{2}-2 \gamma_{k, \theta} \gamma_{k, x}^{2-\tau} \mu_{\theta}\left(2 L_{\theta}^{2}+L_{\theta} L_{x}\right)\right)\left\|x^{k}-x^{*}\right\|^{2} \\
& +3 \gamma_{k, x}^{2}\left\|\nabla_{x} f\left(x^{*} ; \theta^{*}\right)\right\|^{2}+\beta_{k} \gamma_{k, \theta}^{2} v_{\theta}^{2}+\gamma_{k, x}^{2} v_{x}^{2},
\end{aligned}
$$

and $\sigma_{k}=2 \gamma_{k, x}\left(f\left(x^{k} ; \theta^{*}\right)-f\left(x^{*} ; \theta^{*}\right)\right)$. Then, we have

$$
\mathbb{E}\left[\left\|x^{k+1}-y^{k}\right\|^{2} \mid \mathscr{F}_{k}\right]+\beta_{k+1} \mathbb{E}\left[\left\|\theta^{k+1}-\theta^{*}\right\|^{2} \mid \mathscr{F}_{k}\right] \leq\left(1+u_{k}\right)\left(\left\|x^{k}-x^{*}\right\|^{2}+\beta_{k}\left\|\theta^{k}-\theta^{*}\right\|^{2}\right)+\rho_{k}-\sigma_{k} .
$$

By boundedness of $X$ and (A5), we have that $\sum_{k=0}^{\infty} u_{k}<\infty$ and $\sum_{k=0}^{\infty} \rho_{k}<\infty$. By Lemma 3, we obtain that there exists a random variable $V$ such that

$$
\left\|x^{k}-x^{*}\right\|^{2}+\beta_{k}\left\|\theta^{k}-\theta^{*}\right\|^{2} \rightarrow V \quad \text { a.s. } \quad \text { as } k \rightarrow \infty .
$$

and $\sum_{k=0}^{\infty} \sigma_{k}=\sum_{k=0}^{\infty} 2 \gamma_{k, x}\left(f\left(x^{k} ; \theta^{*}\right)-f\left(x^{*} ; \theta^{*}\right)\right)<\infty$.

By (A5), Lemma 2 and (18), we may conclude that $\left\|\theta^{k}-\theta^{*}\right\| \rightarrow 0$ a.s. as $k \rightarrow \infty$. Thus, it follows that $\left\|x^{k}-x^{*}\right\| \rightarrow V$ a.s. as $k \rightarrow \infty$. Since $\sum_{k=0}^{\infty} \gamma_{k, x}=\infty$, we get $\liminf _{k \rightarrow \infty} f\left(x^{k} ; \theta^{*}\right)=f\left(x^{*} ; \theta^{*}\right)$ a.s. as $k \rightarrow \infty$. Since the set $X$ is closed, all accumulation points of $\left\{x^{k}\right\}$ lie in $X$. Furthermore, since $f\left(x^{k} ; \theta^{*}\right) \rightarrow f\left(x^{*} ; \theta^{*}\right)$ along a subsequence a.s. by continuity of $f$ it follows that $\left\{x^{k}\right\}$ has a sequence converging to some random point in $X^{*}$ a.s. Moreover, since $\left\|x^{k}-x^{*}\right\|$ is convergent for any $x^{*} \in X^{*}$ a.s., the entire sequence $\left\{x^{k}\right\}$ converges to some random point in $X^{*}$ a.s.

\section{RATE ANALYSIS}

While the previous section focused on the almost sure convergence of the prescribed SA schemes, a natural question is whether a rate estimate is available. In this section, we show that $O(1 / K)$ rate estimate is derived for an upper bound on the mean squared error in the solution $x_{K}$ when $f\left(\bullet ; \theta^{*}\right)$ is strongly convex in $(\bullet)$ and $K$ represents the number of steps, consistent with the result obtained for stochastic approximation (cf. (Shapiro, Dentcheva, and Ruszczyński 2009)). In addition, it is seen that when the function $f\left(\bullet ; \theta^{*}\right)$ loses strong convexity, an analogous rate estimate is available by using averaging, akin to an approach first employed by Polyak and Juditsky (1992), where longer stepsizes were suggested with consequent averaging of the obtained iterates. Throughout this section, we employ the following notation:

Theorem 6 (Rate estimate for strongly convex $f$ ) Suppose (A1) and (A3) hold. Suppose $\gamma_{x, k}=\lambda_{x} / k$ and $\gamma_{\theta, k}=\lambda_{\theta} / k$ with $\lambda_{x}>1 / \mu_{x}$ and $\lambda_{\theta}>1 /\left(2 \mu_{\theta}\right)$. Let $\mathbb{E}\left[\left\|\nabla f\left(x^{k} ; \theta^{k}\right)+w^{k}\right\|^{2}\right] \leq M^{2}$ and $\mathbb{E}\left[\left\|\nabla g\left(\theta^{k}\right)+v^{k}\right\|^{2}\right] \leq M_{\theta}^{2}$ for all $x^{k} \in X$ and $\theta^{k} \in \Theta$. Let $\left\{x^{k}, \theta^{k}\right\}$ be computed via Algorithm 1. Then, the following hold:

$$
\mathbb{E}\left[\left\|\theta^{k}-\theta^{*}\right\|^{2}\right] \leq \frac{Q_{\theta}\left(\lambda_{\theta}\right)}{k} \text { and } \mathbb{E}\left[\left\|x^{k}-x^{*}\right\|^{2}\right] \leq \frac{Q_{x}\left(\lambda_{x}\right)}{k},
$$

where $Q_{\theta}\left(\lambda_{\theta}\right) \triangleq \max \left\{\lambda_{\theta}^{2} M_{\theta}^{2}\left(2 \mu_{\theta} \lambda_{\theta}-1\right)^{-1}, \mathbb{E}\left[\left\|\theta^{1}-\theta^{*}\right\|^{2}\right]\right\}, Q_{x}\left(\lambda_{x}\right) \triangleq \max \left\{\lambda_{x}^{2} \widetilde{M}^{2}\left(\mu_{x} \lambda_{x}-1\right)^{-1}, \mathbb{E}\left[\left\|x^{1}-x^{*}\right\|^{2}\right]\right\}$, and $\widetilde{M} \triangleq \sqrt{M^{2}+\frac{L_{\theta}^{2} Q_{\theta}\left(\lambda_{\theta}\right)}{\mu_{x} \lambda_{x}}}$. 


\section{Jiang and Shanbhag}

Proof: Suppose $A_{k} \triangleq \frac{1}{2}\left\|x^{k}-x^{*}\right\|^{2}$ and $a_{k} \triangleq \mathbb{E}\left[A_{k}\right]$. Then,

$$
\begin{aligned}
A_{k+1} & =\frac{1}{2}\left\|x^{k+1}-x^{*}\right\|^{2}=\frac{1}{2}\left\|\Pi_{X}\left(x^{k}-\gamma_{x, k}\left(\nabla f\left(x^{k} ; \theta^{k}\right)+w^{k}\right)\right)-\Pi_{X}\left(x^{*}\right)\right\|^{2} \\
& \leq \frac{1}{2}\left\|x^{k}-x^{*}-\gamma_{x, k}\left(\nabla f\left(x^{k} ; \theta^{k}\right)+w^{k}\right)\right\|^{2} \\
& =A_{k}+\frac{1}{2} \gamma_{x, k}^{2}\left\|\nabla f\left(x^{k} ; \theta^{k}\right)+w^{k}\right\|^{2}-\gamma_{x, k}\left(x^{k}-x^{*}\right)^{T}\left(\nabla f\left(x^{k} ; \theta^{k}\right)+w^{k}\right) .
\end{aligned}
$$

Note that $\mathbb{E}\left[\left(x^{k}-x^{*}\right)^{T} w^{k}\right]=\mathbb{E}\left[\mathbb{E}\left[\left(x^{k}-x^{*}\right)^{T} w^{k} \mid \mathscr{F}_{k}\right]\right]=\mathbb{E}\left[\left(x^{k}-x^{*}\right)^{T} \mathbb{E}\left[w^{k} \mid \mathscr{F}_{k}\right]\right]=0$. By taking expectations on both sides of (21) and by invoking the bounds $\mathbb{E}\left[\left\|\nabla f\left(x^{k} ; \theta^{k}\right)+w^{k}\right\|^{2}\right] \leq M^{2}$ and $\mathbb{E}\left[\left\|\nabla g\left(\theta^{k}\right)+v^{k}\right\|^{2}\right] \leq M_{\theta}^{2}$, it follows that

$$
a_{k+1} \leq a_{k}+\frac{1}{2} \gamma_{x, k}^{2} M^{2}-\gamma_{x, k} \mathbb{E}\left[\left(x^{k}-x^{*}\right)^{T} \nabla f\left(x^{k} ; \theta^{k}\right)\right] .
$$

Note that $f(x ; \theta)$ is strongly convex in $x$ with constant $\mu_{x}$ for every $\theta \in \Theta$. Consequently, we have the following expression:

$$
\begin{aligned}
\mathbb{E}\left[\left(x^{k}-x^{*}\right)^{T} \nabla_{x} f\left(x^{k} ; \theta^{k}\right)\right] & =\mathbb{E}\left[\left(x^{k}-x^{*}\right)^{T}\left(\nabla_{x} f\left(x^{k} ; \theta^{k}\right)-\nabla_{x} f\left(x^{*} ; \theta^{k}\right)\right)\right] \\
& +\mathbb{E}\left[\left(x^{k}-x^{*}\right)^{T}\left(\nabla_{x} f\left(x^{*} ; \theta^{k}\right)-\nabla_{x} f\left(x^{*} ; \theta^{*}\right)\right)\right]+\mathbb{E}\left[\left(x^{k}-x^{*}\right)^{T} \nabla_{x} f\left(x^{*} ; \theta^{*}\right)\right] \\
& \geq \mu_{x} \mathbb{E}\left[\left\|x^{k}-x^{*}\right\|^{2}\right]+\mathbb{E}\left[\left(x^{k}-x^{*}\right)^{T}\left(\nabla_{x} f\left(x^{*} ; \theta^{k}\right)-\nabla_{x} f\left(x^{*} ; \theta^{*}\right)\right)\right] .
\end{aligned}
$$

Combining (22) and (23), we get

$$
\begin{aligned}
a_{k+1} & \leq\left(1-2 \gamma_{x, k} \mu_{x}\right) a_{k}+\frac{1}{2} \gamma_{x, k}^{2} M^{2}-\gamma_{x, k} \mathbb{E}\left[\left(x^{k}-x^{*}\right)^{T}\left(\nabla_{x} f\left(x^{*} ; \theta^{k}\right)-\nabla_{x} f\left(x^{*} ; \theta^{*}\right)\right)\right] \\
& \leq\left(1-2 \gamma_{x, k} \mu_{x}\right) a_{k}+\frac{1}{2} \gamma_{x, k}^{2} M^{2}+\frac{1}{2} \gamma_{x, k} \mu_{x} \mathbb{E}\left[\left\|x^{k}-x^{*}\right\|^{2}\right]+\frac{1}{2} \frac{\gamma_{x, k}}{\mu_{x}} \mathbb{E}\left[\left\|\nabla_{x} f\left(x^{*} ; \theta^{k}\right)-\nabla_{x} f\left(x^{*} ; \theta^{*}\right)\right\|^{2}\right] \\
& \leq\left(1-\gamma_{x, k} \mu_{x}\right) a_{k}+\frac{1}{2} \gamma_{x, k}^{2} M^{2}+\frac{1}{2} \frac{\gamma_{x, k}}{\mu_{x}} L_{\theta}^{2} \mathbb{E}\left[\left\|\theta^{k}-\theta^{*}\right\|^{2}\right] .
\end{aligned}
$$

Suppose $\gamma_{\theta, k}=\lambda_{\theta} / k$. Since the function $g(\theta)$ is strongly convex, we can use the standard rate estimate (cf. inequality (5.292) in (Shapiro, Dentcheva, and Ruszczyński 2009)) to get the following

$$
\mathbb{E}\left[\left\|\theta^{k}-\theta^{*}\right\|^{2}\right] \leq \frac{Q_{\theta}\left(\lambda_{\theta}\right)}{k},
$$

where $Q_{\theta}\left(\lambda_{\theta}\right) \triangleq \max \left\{\lambda_{\theta}^{2} M_{\theta}^{2}\left(2 \mu_{\theta} \lambda_{\theta}-1\right)^{-1}, \mathbb{E}\left[\left\|\theta^{1}-\theta^{*}\right\|^{2}\right]\right\}$ with $\lambda_{\theta}>1 /\left(2 \mu_{\theta}\right)$. Suppose $\gamma_{x, k}=\lambda_{x} / k$, allowing us to claim the following:

$$
a_{k+1} \leq\left(1-\frac{\mu_{x} \lambda_{x}}{k}\right) a_{k}+\frac{1}{2} \frac{\lambda_{x}^{2} M^{2}}{k^{2}}+\frac{1}{2} \frac{\lambda_{x} L_{\theta}^{2} Q_{\theta}\left(\lambda_{\theta}\right)}{\mu_{x} k^{2}}=\left(1-\frac{\mu_{x} \lambda_{x}}{k}\right) a_{k}+\frac{1}{2} \frac{\lambda_{x}^{2} \widetilde{M}^{2}}{k^{2}}
$$

where $\widetilde{M}=\sqrt{M^{2}+\frac{L_{\theta}^{2} Q_{\theta}\left(\lambda_{\theta}\right)}{\mu_{x} \lambda_{x}}}$. By assuming that $\lambda_{x}>1 / \mu_{x}$, we obtain that

$$
\mathbb{E}\left[\left\|x^{k}-x^{*}\right\|^{2}\right] \leq \frac{Q_{x}\left(\lambda_{x}\right)}{k}
$$

where $Q_{x}\left(\lambda_{x}\right) \triangleq \max \left\{\lambda_{x}^{2} \widetilde{M}^{2}\left(\mu_{x} \lambda_{x}-1\right)^{-1}, \mathbb{E}\left[\left\|x^{1}-x^{*}\right\|^{2}\right]\right\}$.

A shortcoming of the previous result is the need for strong convexity of $f(x, \theta)$ in $x$ for every $\theta \in \Theta$. In our next result, we weaken this requirement and allow for a merely convex $f$. 


\section{Jiang and Shanbhag}

Assumption 6 (A6) Suppose the following holds in addition to (A4).

(i) For every $x \in X, f(x ; \theta)$ is Lipschitz continuous in $\theta$ with constant $D_{\theta}$.

Theorem 7 (Rate estimates under convexity of $f$ ) Suppose (A3) and (A6) hold. Suppose $\mathbb{E}\left[\| x^{k}-\right.$ $\left.x^{*} \|^{2}\right] \leq M_{x}^{2}, \mathbb{E}\left[\left\|\nabla f\left(x^{k} ; \theta^{k}\right)+w^{k}\right\|^{2}\right] \leq M^{2}$ and $\mathbb{E}\left[\left\|\nabla g\left(\theta^{k}\right)+v^{k}\right\|^{2}\right] \leq M_{\theta}^{2}$ for all $x^{k} \in X$ and $\theta^{k} \in \Theta$. Let $\left\{x^{k}, \theta^{k}\right\}$ be computed via Algorithm 1. For $1 \leq i, t \leq k$, we define $v_{t} \triangleq \frac{\gamma_{x, t}}{\sum_{s=i}^{k} \gamma_{x, s}}, \tilde{x}_{i, k} \triangleq \sum_{t=i}^{k} v_{t} x^{t}$ and $D_{X} \triangleq \max _{x \in X}\left\|x-x^{1}\right\|$. Suppose, for $1 \leq t \leq k, \gamma_{x}$ is defined as

$$
\gamma_{x}=\sqrt{\frac{4 D_{X}^{2}+L_{\theta}^{2} Q_{\theta}\left(\lambda_{\theta}\right)(1+\ln k)}{\left(M^{2}+M_{x}^{2}\right) k}},
$$

where $Q_{\theta}\left(\lambda_{\theta}\right) \triangleq \max \left\{\lambda_{\theta}^{2} M_{\theta}^{2}\left(2 \mu_{\theta} \lambda_{\theta}-1\right)^{-1}, \mathbb{E}\left[\left\|\theta^{1}-\theta^{*}\right\|^{2}\right]\right\}$, and $\gamma_{\theta, k}=\lambda_{\theta} / k$ with $\lambda_{\theta}>1 /\left(2 \mu_{\theta}\right)$. Then the following holds for $1 \leq i \leq k$ :

$$
\mathbb{E}\left[f\left(\tilde{x}_{i, k} ; \theta^{k}\right)-f\left(x^{*} ; \theta^{*}\right)\right] \leq \frac{\sqrt{Q_{\theta}\left(\lambda_{\theta}\right)} D_{\theta}+C_{i, k} \sqrt{B_{k}}}{\sqrt{k}}
$$

where $C_{i, k}=\frac{k}{k-i+1}$ and $B_{k}=\left(4 D_{X}^{2}+L_{\theta}^{2} Q_{\theta}\left(\lambda_{\theta}\right)(1+\ln k)\right)\left(M^{2}+M_{x}^{2}\right)$.

Proof: By using the same notation in Theorem 6, we have from (22) that

$$
\begin{aligned}
a_{k+1} & \leq a_{k}+\frac{1}{2} \gamma_{x, k}^{2} M^{2}-\gamma_{x, k} \mathbb{E}\left[\left(x^{k}-x^{*}\right)^{T} \nabla_{x} f\left(x^{k} ; \theta^{k}\right)\right] \\
& \leq a_{k}+\frac{1}{2} \gamma_{x, k}^{2} M^{2}-\gamma_{x, k} \mathbb{E}\left[\left(x^{k}-x^{*}\right)^{T} \nabla_{x} f\left(x^{k} ; \theta^{*}\right)\right]-\gamma_{x, k} \mathbb{E}\left[\left(x^{k}-x^{*}\right)^{T}\left(\nabla_{x} f\left(x^{k} ; \theta^{k}\right)-\nabla_{x} f\left(x^{k} ; \theta^{*}\right)\right)\right]
\end{aligned}
$$

Note that $f(x ; \theta)$ is convex in $x$ for every $\theta \in \Theta$, allowing us to leverage the gradient inequality.

$$
\mathbb{E}\left[\left(x^{k}-x^{*}\right)^{T} \nabla_{x} f\left(x^{k} ; \theta^{*}\right)\right] \geq \mathbb{E}\left[f\left(x^{k} ; \theta^{*}\right)-f\left(x^{*} ; \theta^{*}\right)\right] .
$$

Combining (25) and (26), we get

$$
a_{k+1} \leq a_{k}+\frac{1}{2} \gamma_{x, k}^{2} M^{2}-\gamma_{x, k} \mathbb{E}\left[f\left(x^{k} ; \theta^{*}\right)-f\left(x^{*} ; \theta^{*}\right)\right]-\gamma_{x, k} \mathbb{E}\left[\left(x^{k}-x^{*}\right)^{T}\left(\nabla_{x} f\left(x^{k} ; \theta^{k}\right)-\nabla_{x} f\left(x^{k} ; \theta^{*}\right)\right)\right] .
$$

This allows for constructing the following bounds:

$$
\begin{aligned}
\gamma_{x, k} \mathbb{E}\left[f\left(x^{k} ; \theta^{*}\right)-f\left(x^{*} ; \theta^{*}\right)\right] & \leq a_{k}-a_{k+1}+\frac{1}{2} \gamma_{x, k}^{2} M^{2}-\gamma_{x, k} \mathbb{E}\left[\left(x^{k}-x^{*}\right)^{T}\left(\nabla_{x} f\left(x^{k} ; \theta^{k}\right)-\nabla_{x} f\left(x^{k} ; \theta^{*}\right)\right)\right] \\
& \leq a_{k}-a_{k+1}+\frac{1}{2} \gamma_{x, k}^{2} M^{2}+\frac{1}{2} \gamma_{x, k}^{2} \mathbb{E}\left[\left\|x^{k}-x^{*}\right\|^{2}\right]+\frac{1}{2} \mathbb{E}\left[\left\|\nabla_{x} f\left(x^{k} ; \theta^{k}\right)-\nabla_{x} f\left(x^{k} ; \theta^{*}\right)\right\|^{2}\right] \\
& \leq a_{k}-a_{k+1}+\frac{1}{2} \gamma_{x, k}^{2} M^{2}+\frac{1}{2} \gamma_{x, k}^{2} M_{x}^{2}+\frac{1}{2} L_{\theta}^{2} \mathbb{E}\left[\left\|\theta^{k}-\theta^{*}\right\|^{2}\right] \\
& \leq a_{k}-a_{k+1}+\frac{1}{2} \gamma_{x, k}^{2}\left(M^{2}+M_{x}^{2}\right)+\frac{1}{2} \frac{L_{\theta}^{2} Q_{\theta}\left(\lambda_{\theta}\right)}{k}
\end{aligned}
$$

where the second inequality follows from the fact that $2 a b \leq a^{2}+b^{2}$, the third inequality follows from the boundedness of $\mathbb{E}\left[\left\|x^{k}-x^{*}\right\|^{2}\right]$ and Lipschitz continuity of $\nabla_{x} f(x ; \theta)$ in $\theta$, and the last inequality follows 


\section{Jiang and Shanbhag}

from (24). As a result, for $1 \leq i \leq k$, we have the following:

$$
\begin{aligned}
\sum_{t=i}^{k} \gamma_{x, t} \mathbb{E}\left[f\left(x^{t} ; \theta^{*}\right)-f\left(x^{*} ; \theta^{*}\right)\right] & \leq \sum_{t=i}^{k}\left(a_{t}-a_{t+1}\right)+\frac{1}{2} \sum_{t=i}^{k} \gamma_{x, t}^{2}\left(M^{2}+M_{x}^{2}\right)+\frac{1}{2} \sum_{t=i}^{k} \frac{L_{\theta}^{2} Q_{\theta}\left(\lambda_{\theta}\right)}{t} \\
& \leq a_{i}+\frac{1}{2} \sum_{t=i}^{k} \gamma_{x, t}^{2}\left(M^{2}+M_{x}^{2}\right)+\frac{1}{2} \sum_{t=i}^{k} \frac{L_{\theta}^{2} Q_{\theta}\left(\lambda_{\theta}\right)}{t} \\
& \leq a_{i}+\frac{1}{2} \sum_{t=i}^{k} \gamma_{x, t}^{2}\left(M^{2}+M_{x}^{2}\right)+\frac{1}{2} L_{\theta}^{2} Q_{\theta}\left(\lambda_{\theta}\right)(1+\ln k) .
\end{aligned}
$$

Next, we define $v_{t} \triangleq \frac{\gamma_{x, t}}{\sum_{s=i}^{k} \gamma_{x, s}}$ and $D_{X} \triangleq \max _{x \in X}\left\|x-x^{1}\right\|$. It follows from invoking these definitions, that

$$
\mathbb{E}\left[\sum_{t=i}^{k} v_{t} f\left(x^{t} ; \theta^{*}\right)-f\left(x^{*} ; \theta^{*}\right)\right] \leq \frac{a_{i}+\frac{1}{2} \sum_{t=i}^{k} \gamma_{x, t}^{2}\left(M^{2}+M_{x}^{2}\right)+\frac{1}{2} L_{\theta}^{2} Q_{\theta}\left(\lambda_{\theta}\right)(1+\ln k)}{\sum_{t=i}^{k} \gamma_{x, t}} .
$$

Next, we consider points given by $\tilde{x}_{i, k} \triangleq \sum_{t=i}^{k} v_{t} x^{t}$. By convexity of $X$, we have that $\tilde{x}_{i, k} \in X$ and by the convexity of $f\left(x ; \theta^{*}\right)$ in $x$, we have $f\left(\tilde{x}_{i, k} ; \theta^{*}\right) \leq \sum_{t=i}^{k} v_{t} f\left(x^{t}\right)$. From (29) and by noting that $a_{1} \leq \frac{1}{2} D_{X}^{2}$ and $a_{i} \leq 2 D_{X}^{2}$ for $i>1$, we obtain the following for $1 \leq i \leq k$

$$
\mathbb{E}\left[f\left(\tilde{x}_{i, k} ; \theta^{*}\right)-f\left(x^{*} ; \theta^{*}\right)\right] \leq \frac{4 D_{X}^{2}+\sum_{t=i}^{k} \gamma_{x, t}^{2}\left(M^{2}+M_{x}^{2}\right)+L_{\theta}^{2} Q_{\theta}\left(\lambda_{\theta}\right)(1+\ln k)}{2 \sum_{t=i}^{k} \gamma_{x, t}} .
$$

Suppose $\gamma_{x, t}=\gamma_{x}$ for $t=1, \ldots, k$. Then, it follows that

$$
\mathbb{E}\left[f\left(\tilde{x}_{1, k} ; \theta^{*}\right)-f\left(x^{*} ; \theta^{*}\right)\right] \leq \frac{4 D_{X}^{2}+k \gamma_{x}^{2}\left(M^{2}+M_{x}^{2}\right)+L_{\theta}^{2} Q_{\theta}\left(\lambda_{\theta}\right)(1+\ln k)}{2 k \gamma_{x}} .
$$

By minimizing the right hand side in $\gamma_{x}>0$, we obtain that

$$
\gamma_{x}=\sqrt{\frac{4 D_{X}^{2}+L_{\theta}^{2} Q_{\theta}\left(\lambda_{\theta}\right)(1+\ln k)}{\left(M^{2}+M_{x}^{2}\right) k}} .
$$

This leads to the following

$$
\mathbb{E}\left[f\left(\tilde{x}_{1, k} ; \theta^{*}\right)-f\left(x^{*} ; \theta^{*}\right)\right] \leq \sqrt{\frac{B_{k}}{k}}, \quad \text { where } B_{k} \triangleq\left(4 D_{X}^{2}+L_{\theta}^{2} Q_{\theta}\left(\lambda_{\theta}\right)(1+\ln k)\right)\left(M^{2}+M_{x}^{2}\right) .
$$

Next, we can also claim that for $1 \leq i \leq k$,

$$
\mathbb{E}\left[f\left(\tilde{x}_{i, k} ; \theta^{*}\right)-f\left(x^{*} ; \theta^{*}\right)\right] \leq C_{i, k} \sqrt{\frac{B_{k}}{k}},
$$

where $C_{i, k}=\frac{k}{k-i+1}$. Thus, by the Lipschitz continuity of $f(x ; \theta)$ in $\theta,(24)$, and (33), we have

$$
\begin{aligned}
\mathbb{E}\left[f\left(\tilde{x}_{i, k} ; \theta^{k}\right)-f\left(x^{*} ; \theta^{*}\right)\right] & \leq \mathbb{E}\left[f\left(\tilde{x}_{i, k} ; \theta^{k}\right)-f\left(\tilde{x}_{i, k} ; \theta^{*}\right)\right]+\mathbb{E}\left[f\left(\tilde{x}_{i, k} ; \theta^{*}\right)-f\left(x^{*} ; \theta^{*}\right)\right] \\
& \leq D_{\theta} \mathbb{E}\left[\left\|\theta^{k}-\theta^{*}\right\|\right]+\mathbb{E}\left[f\left(\tilde{x}_{i, k} ; \theta^{*}\right)-f\left(x^{*} ; \theta^{*}\right)\right] \\
& \leq \frac{\sqrt{Q_{\theta}\left(\lambda_{\theta}\right)} D_{\theta}}{\sqrt{k}}+\mathbb{E}\left[f\left(\tilde{x}_{i, k} ; \theta^{*}\right)-f\left(x^{*} ; \theta^{*}\right)\right] \leq \frac{\sqrt{Q_{\theta}\left(\lambda_{\theta}\right)} D_{\theta}+C_{i, k} \sqrt{B_{k}}}{\sqrt{k}} .
\end{aligned}
$$

In effect, in the context of learning and optimization, the averaging approach leads to a complexity bound given loosely by $O\left(1 / \sqrt{\frac{k}{\ln (k)}}\right)$, while the standard bound leads to $O\left(\frac{1}{\sqrt{k}}\right)$. While it is not surprising that the requirement to learn $\theta^{*}$ imposes a degradation, it appears that this degradation is not severe. 
Jiang and Shanbhag

\section{CONCLUDING REMARKS}

We consider a problem of solving a stochastic optimization problem in which the objective is parameterized by a vector that can be learnt by solving a distinct stochastic optimization problem. In both convex and merely convex regimes, we develop a set of coupled stochastic approximation schemes which produces a sequence of iterates that are shown to converge to the solution and unknown parameter in an almost sure sense. Additionally, we provide rate estimates for the prescribed schemes in both strongly convex and convex regimes. From a rate standpoint, it is seen that the additional learning component does not affect the rate for strongly convex problems but does so for merely convex problems. Future research is expected to develop along multiple directions, including an extension to variational regimes, the examination of the relationship to regret-based online optimization, and the development of a set of supporting numerics.

\section{REFERENCES}

Astrom, K. J., and B. Wittenmark. 1994. Adaptive Control. 2nd ed. Boston, MA, USA: Addison-Wesley Longman Publishing Co., Inc.

Cooper, W. L., T. Homem-de Mello, and A. J. Kleywegt. 2006, September. "Models of the Spiral-Down Effect in Revenue Management". Oper. Res. 54:968-987.

Gittins, J. C. 1989. Multi-armed bandit allocation indices. Wiley-Interscience Series in Systems and Optimization, Chichester: John Wiley \& Sons, Ltd.

Hastie, T., R. Tibshirani, and J. H. Friedman. 2001. The elements of statistical learning: data mining, inference, and prediction: with 200 full-color illustrations. New York: Springer-Verlag.

Jiang, H., U. V. Shanbhag, and S. P. Meyn. 2011. "Learning equilibria in constrained Nash-Cournot games with misspecified demand functions". In Proceedings of the IEEE Conference on Decision and Control (CDC-ECE), 1018-1023.

Koshal, J., A. Nedic, and U. V. Shanbhag. 2013. "Regularized Iterative Stochastic Approximation Methods for Stochastic Variational Inequality Problems”. IEEE Trans. Automat. Contr. 58 (3): 594-609.

Moore, K. L. 1993. Iterative Learning Control for Deterministic Systems. Springer-Verlag Series on Advances in Industrial Control. London: Springer-Verlag.

Polyak, B. T. 1987. Introduction to optimization. New York: Optimization Software, Inc.

Polyak, B. T., and A. B. Juditsky. 1992. "Acceleration of stochastic approximation by averaging". SIAM J. Control Optim. 30 (4): 838-855.

Shapiro, A., D. Dentcheva, and A. Ruszczyński. 2009. Lectures on stochastic programming, Volume 9 of MPS/SIAM Series on Optimization. Philadelphia, PA: SIAM. Modeling and theory.

\section{AUTHOR BIOGRAPHIES}

HAO JIANG is a Ph.D. student in the Department of Industrial and Enterprise Systems Engineering at University of Illinois at Urbana-Champaign. His research interests include learning, variational inequalities and game theory. His email address is jiang23@illinois.edu.

UDAY V. SHANBHAG is an Associate Professor in the Harold and Inge Marcus Department of Industrial and Manufacturing Engineering at Penn. State University. He received his Ph.D. degree in operations research from the Department of Management Science and Engineering, Stanford University, Stanford, CA, in 2006. His interests lies in the development of analytical and algorithmic tools in the context of optimization and variational problems, in regimes complicated by uncertainty, dynamics and nonsmoothness. His email address is udaybag@psu.edu and his web page is http://www2.ie.psu.edu/shanbhag/personal/index.htm. 